\title{
A Hybrid Multiobjective Discrete Particle Swarm Optimization Algorithm for Cooperative Air Combat DWTA
}

\author{
Guang Peng, Yangwang Fang, Shaohua Chen, Weishi Peng, and Dandan Yang \\ School of Aeronautics and Astronautics Engineering, Air Force Engineering University, Xian, Shaanxi 710038, China \\ Correspondence should be addressed to Guang Peng; pg1445334307@163.com
}

Received 30 December 2016; Accepted 20 March 2017; Published 11 April 2017

Academic Editor: Maoguo Gong

Copyright (C) 2017 Guang Peng et al. This is an open access article distributed under the Creative Commons Attribution License, which permits unrestricted use, distribution, and reproduction in any medium, provided the original work is properly cited.

\begin{abstract}
A hybrid multiobjective discrete particle swarm optimization (HMODPSO) algorithm is proposed to solve cooperative air combat dynamic weapon target assignment (DWTA). First, based on the threshold of damage probability and time window constraints, a new cooperative air combat DWTA multiobjective optimization model is presented, which employs the maximum of the target damage efficiency and minimum of ammunition consumption as two competitive objective functions. Second, in order to tackle the DWTA problem, a mixed MODPSO and neighborhood search algorithm is proposed. Furthermore, the repairing operator is introduced into the mixed algorithm, which not only can repair infeasible solutions but also can improve the quality of feasible solutions. Besides, the Cauchy mutation is adopted to keep the diversity of the Pareto optimal solutions. Finally, a typical two-stage DWTA scenario is performed by HMODPSO and compared with three other state-of-the-art algorithms. Simulation results verify the effectiveness of the new model and the superiority of the proposed algorithm.
\end{abstract}

\section{Introduction}

The weapon target assignment (WTA) is a typical NPcomplete constrained combinatorial optimization problem [1], which can be classified into two categories: static WTA (SWTA) and dynamic WTA (DWTA) [2, 3]. In SWTA, all the weapons attack targets in a single stage. In contrast, DWTA is much more complicated than SWTA, which takes the time window and resource constraints into account [4]. Besides, DWTA needs to deal with the new incoming targets and assesses the outcome of each engagement.

Most of the previous researches on WTA are focused on SWTA [4]. However, DWTA has begun to gain more attention of researchers since it was put forward by Hosein and Athans in 1990 [3]. Cai et al. [4] provided a survey of the research on DWTA problem and introduced some basic concepts on DWTA. Khosla [5] proposed a hybrid approach, which combines genetic algorithm (GA) with simulated annealing (SA) to solve a target-based DWTA problem. Liu et al. [6] analyzed the time and space restriction of the mathematical model of DWTA and proposed an adaptive memetic algorithm to obtain the suboptimum solution step by step. Chen et al. [7] established a generic asset-based DWTA model which incorporates four categories of constraints, namely, capability constraints, strategy constraints, resource constraints, and engagement feasibility constraints. Based on the asset-based DWTA model, Xin et al. [8] proposed a new technique for constraint handling. Moreover, Xin et al. [9] proposed an efficient rule-based heuristic, which uses the domain knowledge of DWTA in the form of three crucial rules, to solve asset-based DWTA problems. The proposed method has obvious advantage over the Monte Carlo method (MCM) with regards to solution quality and computation time. Wang et al. [10] applied intuitionistic fuzzy entropy of discrete particle swarm optimization (IFDPSO) algorithm to solve DWTA problem. Wang et al. [11] firstly established a multicombat step DWTA game model of UAV aerial combat and then presented a clonal selection optimization algorithm to solve the model.

However, the above WTA problems are focused on one objective (i.e., operational effects), ignoring the operational cost, while in actual combat situations, apart from considering the maximum of the damage to targets, the ammunition consumption should be also taken into account. Clearly, the two competitive objectives are conflicting, which implies that the WTA problem is a multiobjective optimization problem. 


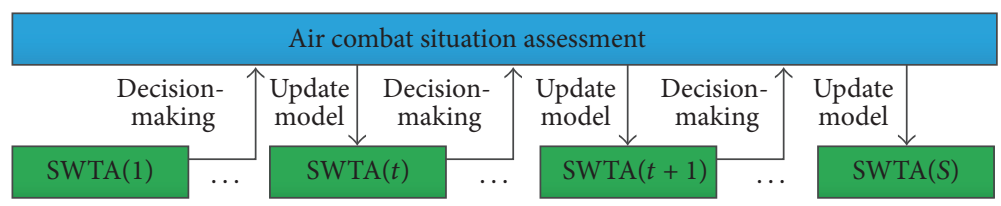

FIGURE 1: The schematic diagram of cooperative air combat DWTA model.

Up to now, there have been several studies on multiobjective optimization for DWTA problems. Liu et al. [12] and Zhou et al. [13] proposed an improved MOPSO algorithm to solve the multiobjective programming model of SWTA, respectively. Lötter and Van Vuuren [14] used NSGA-II to solve a triobjective DWTA model for surface-based air defense. Li et al. [15, 16] established deterministic and uncertain multiobjective optimization models of multistage WTA (MWTA) problem and modified two multiobjective optimizers, NSGA-II and MOEA/D, by adding an adaptive mechanism for solving the NWTA models. However, MOPSO easily falls into the local optimum; NSGA-II and MOEA/D have complex computation for DWTA. DWTA problem has higher requirements for the real-time performance and the convergence. In order to meet the real-time performance and the convergence accuracy simultaneously, this paper proposed an efficient HMODPSO algorithm to solve the DWTA multiobjective optimization problem. The proposed HMODPSO algorithm can generate obviously better DWTA decisions without the cost of overmuch extra computation time, which can improve the cooperative air combat effectiveness.

The rest of this paper is organized as follows. In Section 2, the cooperative air combat DWTA multiobjective optimization model based on the threshold of damage probability is formulated. Section 3 presents the structure of HMODPSO algorithm. The simulation results based on the proposed algorithm for a typical two-stage DWTA scenario are discussed in Section 4, and also the comparisons with three other state-of-the-art algorithms are conducted in this section. Conclusions and future work will be drawn in Section 5.

\section{The Cooperative Air Combat Multiobjective Optimization Model for DWTA}

Definition 1 (time window of target). The time window of target $\left(t^{T}\right)$ is the exposure time of a target which the weapon can attack efficiently.

Definition 2 (time window of algorithm). The time window of algorithm $\left(t^{A}\right)$ is the running time for solving the DWTA.

A complete cooperative air combat is a multistage offensive and defensive process. So the DWTA model can be regarded as the repetition of the SWTA model with the damage assessment. There are at most $S$ stages of DWTA, which means no targets or weapons left after final stage assignment. In the cooperative air combat DWTA model, the "shoot-look-shoot" engagement policy is adopted. When entering the $t+1$ stage, it is necessary to determine a set of alive targets and remaining weapons. Therefore, it needs to observe the outcome of the $t$ stage engagement and reformulate air combat situation assessment. The schematic diagram of cooperative air combat DWTA model is shown in Figure 1.

2.1. The DWTA Multiobjective Optimization Model. Assuming in the cooperative air combat that there are $F$ flights in blue formation and each flight carries $M_{f}(f=1,2, \ldots, F)$ missiles, the total number of weapons is $M=\sum_{f=1}^{F} M_{f}$. At a certain time, the formation detects $N$ targets, which can be attacked by the weapons. $p_{i j}$ is the damage probability that the $i$-th missile attacks $j$-th target. $w_{j}$ is the threat value of target $j$. Obviously, $p_{i j}$ and $w_{j}$ can be obtained by $\mathrm{C}^{4}$ I system according to the weapons' performance and air combat situation.

In order to describe the DWTA problem, a Boolean type decision matrix is introduced:

$$
X=\left[\begin{array}{cccc}
x_{11} & x_{12} & \cdots & x_{1 N} \\
x_{21} & x_{22} & \cdots & x_{2 N} \\
& & \vdots & \\
& & \cdots & \\
x_{M 1} & x_{M 2} & \cdots & x_{M N}
\end{array}\right],
$$

where $x_{i j}=1$ if the weapon $i$ is assigned to the target $j$ and $x_{i j}=0$ otherwise. After all the weapons attack the target $j$ cooperatively, the joint damage probability of the target $j$ can be expressed as

$$
P_{j}=1-\prod_{i=1}^{M}\left(1-p_{i j}\right)^{x_{i j}} .
$$

In order to avoid wasting weapon resources in the single WTA optimization model, we define the maximum of the target damage efficiency and minimum of using weapon units as two objective functions. Additionally, aiming at targets with different threat values, the new model should guarantee the threshold of damage probability of each target. Hence, the formulation of the objective functions for the stage $t$ is constructed:

$$
\begin{aligned}
& \max f(t)=\sum_{j=1}^{N(t)} w_{j}(t)\left[1-\prod_{i=1}^{M(t)}\left(1-p_{i j}(t)\right)^{x_{i j}(t)}\right] \\
& \min g(t)=\sum_{j=1}^{N(t)} \sum_{i=1}^{M(t)} x_{i j}(t),
\end{aligned}
$$

where $t$ is the stage index; $M(t)$ and $N(t)$ are the numbers of existing weapons and targets at the stage $t$, respectively; $p_{i j}(t)$ 
is the damage probability that the $i$-th missile attacks the $j$-th target at the stage $t ; x_{i j}(t)$ is the Boolean type decision variable at the stage $t$.

The following three categories of constraints are incorporated in the cooperative air combat DWTA multiobjective optimization model:

$$
\begin{array}{r}
P_{j}(t) \geq P_{d j}(t), \\
\forall t \in\{1,2, \ldots, S\}, \forall j \in\{1,2, \ldots, N\} \\
\sum_{j=1}^{N(t)} x_{i j}(t) \leq 1, \quad \forall t \in\{1,2, \ldots, S\}, \forall i \in\{1,2, \ldots, M\} \\
t_{j}^{T}(t) \geq t^{A}(t), \\
\forall t \in\{1,2, \ldots, S\}, \forall j \in(1,2, \ldots, N) .
\end{array}
$$

Constraint set (4) represents the threshold of damage probability of each target, the value of which depends on the decision-makers or the command system based on the current air combat situation. If one of the targets does not satisfy the threshold, the weapon target assignment is regarded as invalid assignment. Constraint set (5) reflects the capability of weapons attacking targets at the same time. In fact, a missile can only shoot one target each time. Constraint set (6) is very important for DWTA model, which takes the time windows of target and algorithm into account, influencing the engagement feasibility of weapons. In this case, it makes particularly high requirements for operational efficiency of the algorithm.

2.2. Constraints Handling. The DWTA multiobjective optimization model is a typical constrained nonlinear combinational optimization problem. To ensure the feasibility of solutions generated by the proposed algorithm, we make some preliminary treatments to constraints (4)-(6). First, we utilize the penalty function method to handle the constraint set (4). The function is defined as

$$
h_{j}(t)=\min \left(0, P_{j}(t)-P_{d j}(t)\right) \text {. }
$$

For constraint set (5), it can be satisfied by solution encoding. And the constraint set (6) is satisfied by adopting the proposed algorithm.

Above all, the multiobjective optimization model for the cooperative air combat DWTA problem aforementioned can be formulated as

$$
\begin{aligned}
& \max \quad f(t) \\
& =\sum_{j=1}^{N(t)} w_{j}(t)\left[1-\prod_{i=1}^{M(t)}\left(1-p_{i j}(t)\right)^{x_{i j}(t)}\right] \\
& -\sigma \sum_{j=1}^{N} h_{j}(t)^{2} \\
& \min \quad g(t)=\sum_{j=1}^{N(t)} \sum_{i=1}^{M(t)} x_{i j}(t),
\end{aligned}
$$

where $\sigma$ is the penalty parameter and $\sigma=1000$ is selected in this paper.

\section{HMODPSO Algorithm for DWTA}

3.1. Particle Position Encoding. Decimal encoding for particles is adopted in this paper. The length of the particle position encoding (denoted by $D$ ) is the total number of weapons. Each weapon is treated as a dimension of particle position encoding, and the value of each dimension indicates the number of the targets to which the weapon is assigned. Figure 2 provides an example to explain the encoding method $(M=6, N=4, t=1)$, and $X$ is the corresponding $0-1$ decision matrix. Obviously, such a particle position encoding method can guarantee that all the generated solutions satisfy the constraint set (5).

3.2. The Leader Particle Selecting. In PSO [17], each particle in the swarm corresponds to a potential solution of the optimization problem. In a $D$-dimensional search space, each particle has a position $X_{i}(k)=\left[X_{i, 1}(k), X_{i, 2}(k), \ldots, \mathrm{X}_{i, D}(k)\right]$ and a velocity $V_{i}(k)=\left[V_{i, 1}(k), V_{i, 2}(k), \ldots, V_{i, D}(k)\right]$. During the movement, the personal best position (Pbest) for each particle is recorded as $P_{i}(k)=\left[P_{i, 1}(k), P_{i, 2}(k), \ldots, P_{i, D}(k)\right]$, and the global best particle (Gbest) of the swarm is referred to as $G(k)=\left[G_{1}(k), G_{2}(k), \ldots, G_{D}(k)\right]$. For specific DWTA problem, the velocity and position of the particle are updated by the following equations, respectively:

$$
\begin{aligned}
& V_{i j}(k+1) \\
& =w V_{i j}(k)+c_{1} r_{1}\left(P_{i j}(k)-X_{i j}(k)\right) \\
& \quad+c_{2} r_{2}\left(G_{j}(k)-X_{i j}(k)\right) \\
& X_{i j}(k+1)=\operatorname{round}\left(X_{i j}(k)+V_{i j}(k+1)\right) \\
& V_{i j}(k+1) \longrightarrow \max \left(\min \left(V_{i j}(k+1), V_{\max }\right), V_{\min }\right) \\
& X_{i j}(k+1)=\max \left(\min \left(X_{i j}(k+1), X_{\max }\right), X_{\min }\right),
\end{aligned}
$$

where $i$ represents the $i$-th particle of the swarm, $j$ represents the $j$-th dimension in the search space, $k$ is the number of current iterations, $w$ is the inertia weight, $c_{1}, c_{2}$ are the acceleration coefficients, and $r_{1}, r_{2} \in[0,1]$ are uniformly distributed random variables. $X_{\max }$ and $X_{\min }$ are the lower and upper boundaries of the position, respectively; $V_{\max }$ and $V_{\min }$ are the lower and upper boundaries of the velocity, respectively. round() is said to be an integer operator. In MOPSO, selecting the global best position or the leader (Gbest) randomly from the external archive is a popular way [12]. However, the roulette method may damage evolution direction of the particles. Considering the weakness, a new method of selecting the leader particle is presented. First, the square root distance (SRD) [18] of particles $X_{1}$ and $X_{2}$ is defined as follows:

$$
\operatorname{SRD}\left(X_{1}, X_{2}\right)=\sum_{i=1}^{m} \sqrt{\left|f_{i}\left(X_{1}\right)-f_{i}\left(X_{2}\right)\right|},
$$




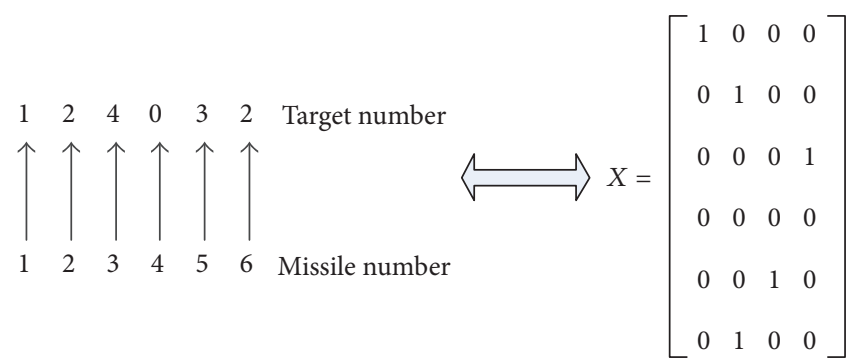

Figure 2: Particle position encoding.

where $m$ represents the number of the objective functions. During the process of iteration, first, calculate all the particles' SRD with the nondominated solutions in the external archive; then, select the nondominated solution which has the minimum SRD as the leader particle; that is,

$$
\min \left(\sum_{i=1}^{m} \sqrt{\left|f_{i}\left(Y_{1}\right)-f_{i}(X)\right|}, \ldots, \sum_{i=1}^{L} \sqrt{\left|f_{i}\left(Y_{L}\right)-f_{i}(X)\right|}\right),
$$

where $\left\{Y_{1}, Y_{2}, \ldots, Y_{L}\right\}$ represents the $L$ nondominated solutions in the external archive and $X$ represents the current position of particle. Particles can choose closer nondominated solutions as their leader particles through the new method, which can help the algorithm overcome the uncertainty of random selection and improve the convergence accuracy.

3.3. Repairing Operator. Clearly, unfeasible solutions which do not satisfy the constraint set (4) may be generated for solving the DWTA. To cope with this issue, a repairing operator is introduced [19], which includes two parts.

(1) Deleting the Redundant Allocation. First, select the unfeasible solutions and mark the targets satisfying the threshold of the damage probability. Then, try to delete the missile attacking the target with minimum damage probability. After deleting, if the target still satisfies the threshold, confirm the deletion; otherwise, retain the missile.

(2) Supplementing the Insufficient Allocation. First, select the targets which do not satisfy threshold of damage probability. Then mark the target with the minimum joint damage probability, and select one missile attacking the target with the maximum damage probability. Repeat the operation until no weapons are left or all the targets satisfy the threshold of damage probability.

The corresponding pseudocode of the repairing operator is shown in Algorithm 1.

3.4. Cauchy Mutation Operator. In order to further maintain the diversity of particles, the Cauchy mutation [20] is introduced into HMODPSO algorithm. When the current particle is dominated by Pbest, the Cauchy mutation is applied to

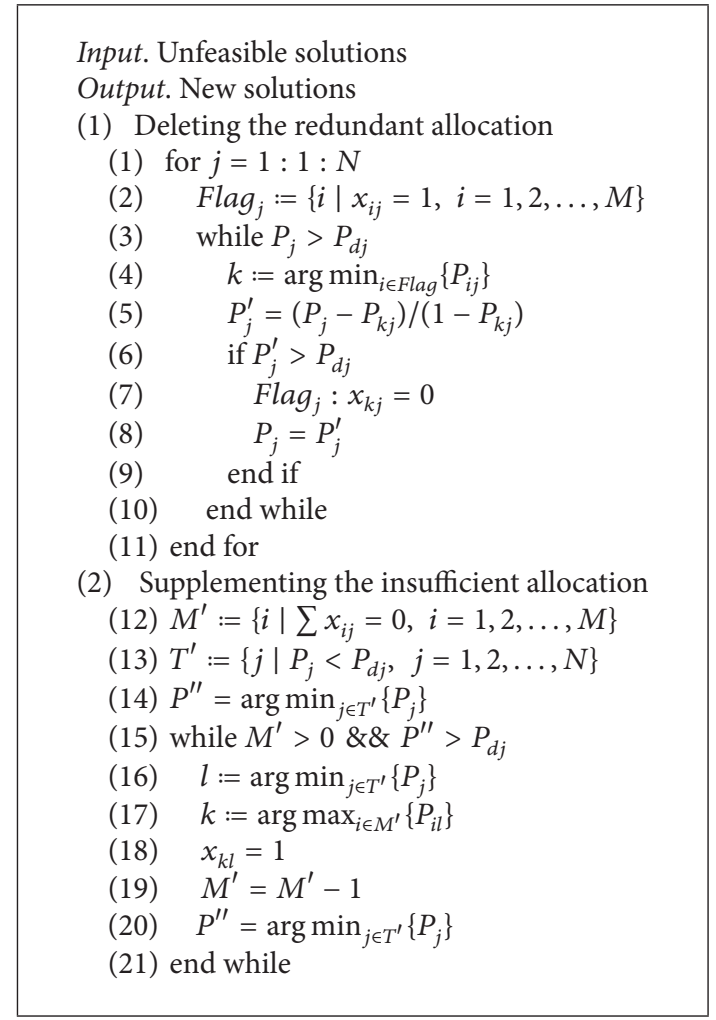

Algorithm 1: The pseudocode of the repairing operator.

disturb the current particle. The Cauchy mutation operator is defined as

$$
\begin{aligned}
& X_{i j} \\
& = \begin{cases}X_{i j}+\operatorname{round}\left(\left(X_{\max }-X_{\min }\right) \times \operatorname{Cauchy}(0,1)\right), & \text { rand } \leq p_{b} \\
X_{i j}, & \text { rand }>p_{b}\end{cases} \\
& \text { Cauchy }(0,1)=\tan ((\operatorname{rand}-0.5) \times \pi) \\
& X_{i j}= \begin{cases}X_{\min }, & X_{i j}<X_{\min } \\
X_{\max }, & X_{i j}>X_{\max } \\
X_{i j}, & \text { elsewhile, }\end{cases}
\end{aligned}
$$




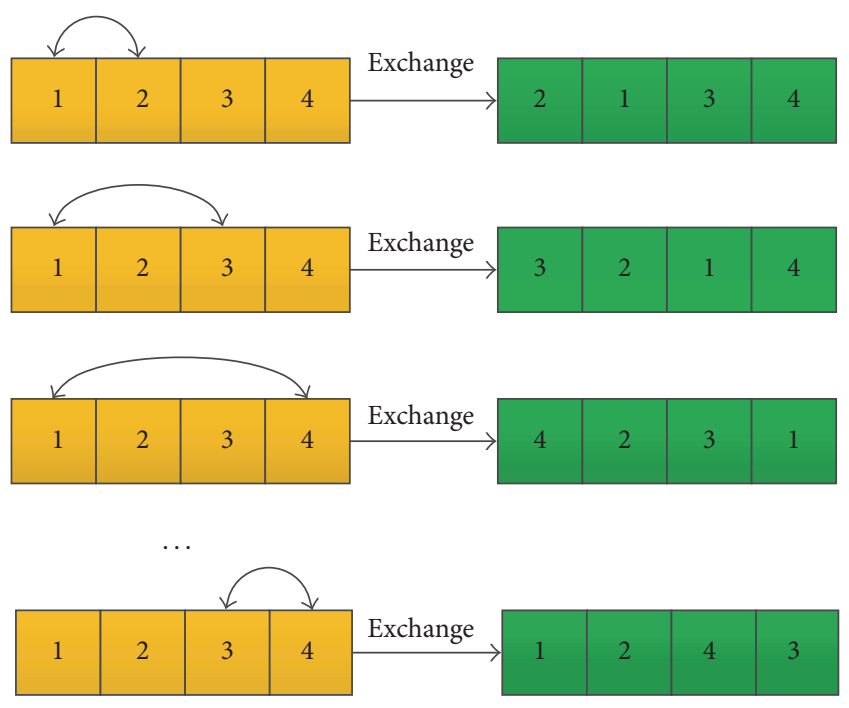

FIGURE 3: Neighborhood exchange criterion.

where rand $\epsilon[0,1]$ is a uniformly distributed random value, Cauchy $(0,1)$ is a standard Cauchy-distributed random value, and the adapting mutation rate $P_{b}$ is computed as

$$
P_{b}=1-\sqrt{\frac{t}{\max I t}} .
$$

3.5. Neighborhood Searching Operator. Neighborhood search (NS) algorithm begins with an initial solution and searches the better solution in its neighborhood range [21]. At present, define a solution $X=\{1,2,3,4\}$, and a new vector can be obtained by exchanging any two positions of the solution. A collection of all the new vectors is referred to as exchange neighborhood shown in Figure 3.

NS algorithm is usually carried out at the end of the global search for individual local optimization, which can improve the convergence accuracy of algorithm. In MOPSO, the nondominated solutions in the external archive are regarded as leader particles to guide the swarm to fly. Additionally, the nondominated solutions are evolved into the Pareto optimal solutions set. In view of the particularity of the external archive, NS algorithm is introduced into the external archive. This paper proposed three kinds of operations: NS-1, NS-2, and NS-3. The procedures are described as follows.

For NS-1, do the following steps.

Step 1. Take each nondominated solution in the external archive as the initial solution.

Step 2. Exchange randomly two positions of the initial solution to get a new solution. If the new solution is better than the initial solution, then replace it with the new solution; otherwise, keep the initial solution.

For NS-2, do the following steps.

Step 1. Take each nondominated solution in the external archive as the initial solution.

\section{NS-1}

(1) for $i=1: 1: L$

(2) $N X=$ crossover $(X, X)$

(3) evaluatefitness $(N X)$

(4) $X=$ Compare $(X, N X)$

(5) end for

NS-2

(1) for $i=1: 1: L$

(2) for $j=1: 1: D-1$

(3) for $k=j+1: 1: D$

(4) $\quad N X=$ exchange $(X(j, k))$

(5) evaluatefitness $(N X)$

(6) end for

(7) end for

(8) $X=$ Compare $(X, \max (N X))$

(9) end for

NS-3

(1) for $i=1: 1: L$

(2) for $j=1: 1: D-1$

(3) for $k=j+1: 1: D$

(4) $\quad N X=$ exchange $(X(j, k))$

(5) evaluatefitness $(N X)$

(6) $X=$ Compare $(X, N X)$

(7) end for

(8) end for

(9) end for

Algorithm 2: The pseudocode of the local search operations.

Step 2. Select the best solution from the neighborhood range of the initial solution.

Step 3. If the best solution is better than the initial solution, then replace it with the new solution; otherwise, keep the initial solution.

For NS-3, do the following steps.

Step 1. Take each nondominated solution in the external archive as the initial solution.

Step 2. Exchange two positions of the initial solution in sequence to get a new solution; if the new solution is better than the initial solution, replace it with the new solution; then serve the new solution as the initial solution and repeat the above operation; otherwise, keep the initial solution.

The corresponding pseudocode of the local search operations is shown in Algorithm 2.

3.6. The Procedure of HMODPSO Algorithm. Summarizing the above procedures, we obtain the following pseudocode of the HMODPSO algorithm in Algorithm 3, where CheckBoundaries validates the particles to search in the solution space and max It is the maximum iterations. Furthermore, use the crowding distance sorting strategy [22] to maintain the external archive. Figure 4 illustrates the entire flowchart of HMODPSO. 


\section{Simulations and Results}

In the experimental scenario, set $F=5, M_{f}=4(f=$ $1,2,3,4,5)$, and $N=10$, and obtain $M=20$. When $t=1$, the time window of each target $t_{j}^{T}(j=1,2, \ldots, 10)$ is $[8.3,11.4,16.2,13.8,20.1,25.4,19.6,13.5,17.2,22.8]$; and each target's threat coefficient $w_{j}(j=1,2, \ldots, 10)$ is $[0.6,0.7,0.3,0.5,0.6,0.35,0.65,0.55,0.4,0.75]$. The damage probability threshold of each target can be set to 0.9 . The weapon's damage probability $p_{i j}(i=1,2, \ldots, 20, j=$ $1,2, \ldots, 10)$ is

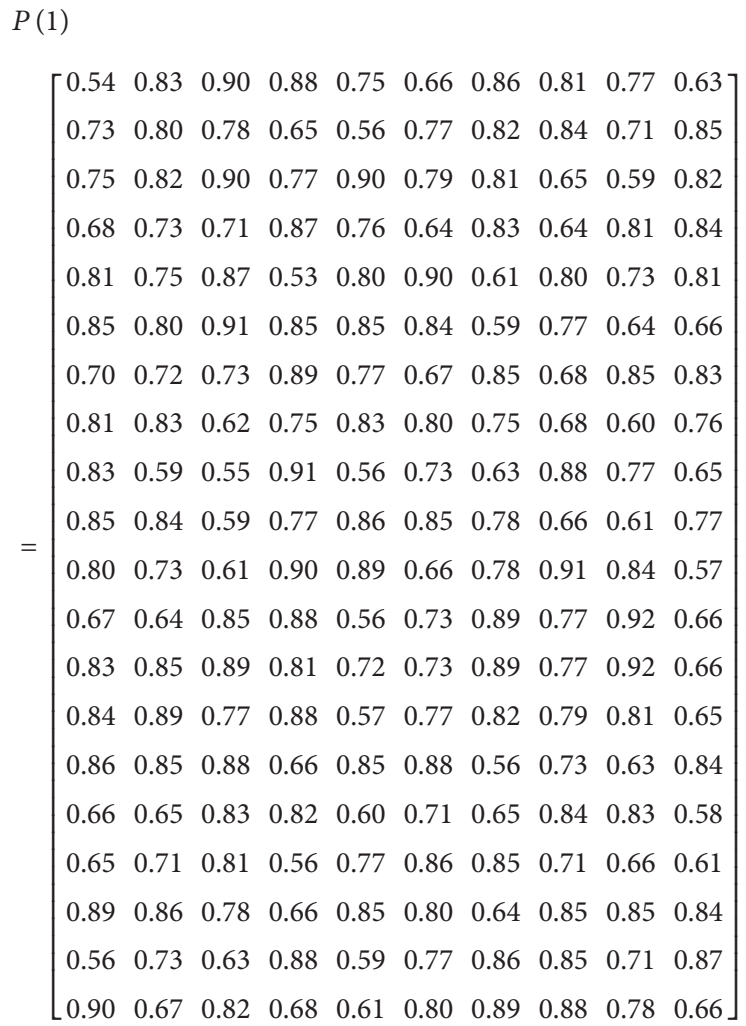

To verify the efficiency of HMODPSO algorithm, three different kinds of algorithms were proposed: HMODPSO1 (adopting NS-1 operation), HMODPSO-2 (adopting NS-2 operation), and HMODPSO-3 (adopting NS-3 operation). At the same time, compare the proposed algorithms with NSGAII [23], MODPSO [12], and MODPSO-GSA [24] to show their potential competences. In the experiment, population size is 60, 100 iterations are carried out, the external archive size is chosen as 30, the crossover probability of NSGA-II is 0.8 , and the mutation rate of NSGA-II is 0.1. All the simulations were performed under the same environment (Matlab) on Intel Core i5-4590 3.3 GHz CPU with 4 GB RAM.

The Pareto fronts produced by six algorithms are shown in Figure 5; it can be seen that all the solutions using HMODPSO are better than the other three algorithms. MODPSO-GSA can also get good solutions. However, MODPSO and NSGA-II easily fall into the local optimum. The different Pareto fronts are compared in Figure 6.
For each algorithm, 30 independent runs were executed. The computational time of algorithm is represented by $T$. The average results of the Pareto optimal solutions attained by six algorithms are shown in Table 1.

Owing to the time window constraint of the DWTA multiobjective optimization model, the computational time of algorithm must satisfy requirements of the targets' time window. From Table 1, NSGA-II, HMODPSO-2, and HMODPSO-3 algorithms do not meet the requirements. MODPSO has the fastest convergence speed, but it easily falls into the local optimum. Compared with MODPSO-GSA, HMODPSO-1 has better comprehensive performance. So, in the $t=1$ stage, the assignment $(f=5.0524, g=13$ ) which was obtained once by HMODPSO-1 can be selected for the engagement. Figure 7 shows the damage probability of each target and the scheme of the assignment $(f=5.0524, g=$ 13).

After the first engagement, the parameters $M(t+1), N(t+$ $1), w_{j}(t+1), p_{i j}(t+1)$, and $t_{j}^{T}(t+1)$ need to be updated in the next stage according to the target damage assessment and the new air combat situation. Assume that, in the $t=2$ stage, $M(2)=7$ and $N(2)=4$. The damage probability threshold of each target can be set to 0.9 . The time window of each target $t_{j}^{T}(2)$ is $[6.5,8.1,5.9,0.2]$; and each target's threat coefficient $w_{j}(2)$ is $[0.6,0.8,0.5,0.7]$. The weapon's damage probability $p_{i j}(2)$ is

$$
P(2)=\left[\begin{array}{llll}
0.65 & 0.74 & 0.85 & 0.81 \\
0.72 & 0.77 & 0.92 & 0.59 \\
0.86 & 0.63 & 0.71 & 0.78 \\
0.53 & 0.80 & 0.73 & 0.65 \\
0.90 & 0.62 & 0.79 & 0.74 \\
0.82 & 0.91 & 0.75 & 0.84 \\
0.56 & 0.65 & 0.73 & 0.94
\end{array}\right] .
$$

Since $t_{4}^{T}(2)=0.2 \mathrm{~s}$ is so small that it is difficult to satisfy the time window of the special target, a new priority selecting method is efficiently adopted to deal with several special targets whose time windows do not satisfy the time window constraint of the proposed algorithm. From the existing weapons at a certain stage, select the missile with the maximum damage probability to attack the special target; repeat this operation until the special target's joint damage probability satisfies the threshold. As seen from $P(2)$, the 7 th missile attacking the target can satisfy the threshold of damage probability. The assignment of the remaining targets can be solved by HMODPSO-1 algorithm. Figure 8 shows the Pareto front.

HMODPSO-1 was independently run for 30 times. The average results of the Pareto optimal solutions attained by HMODPSO-1 are shown in Table 2.

In the $t=2$ stage, the assignment $(f=1.7280, g=3)$ can be selected for the engagement. Figure 9 shows the damage probability of each target and the scheme of the assignment $(f=1.7280, g=3)$. HMODPSO-1 can efficiently solve the 


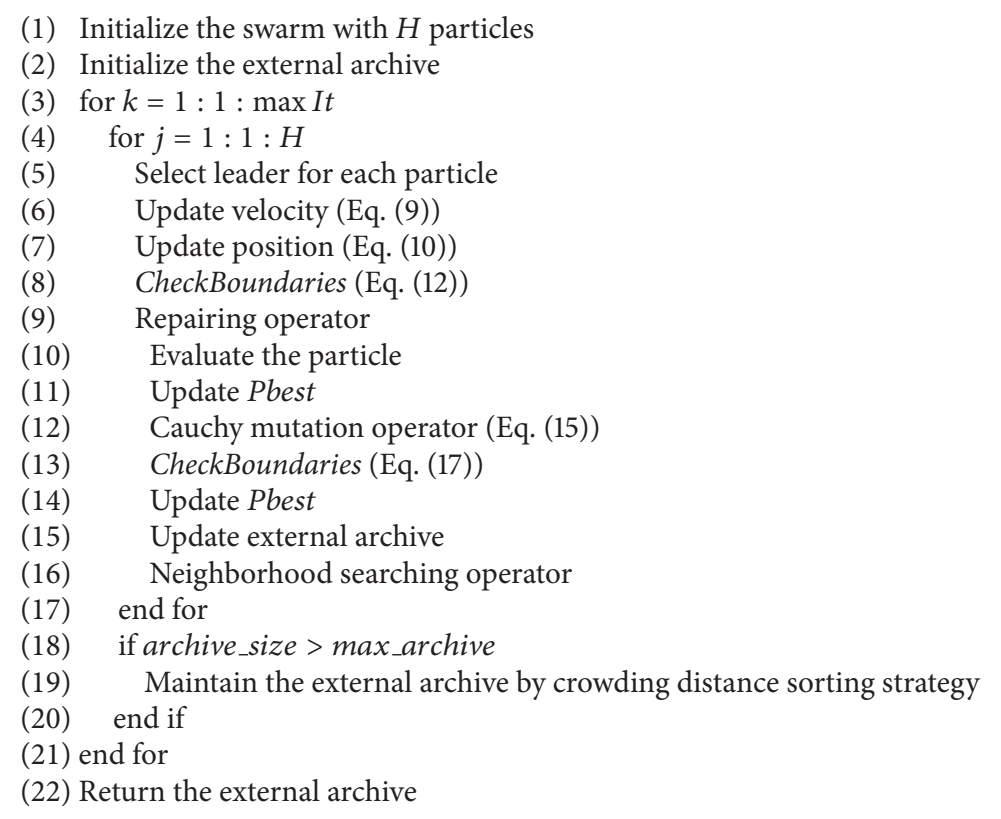

Algorithm 3: The pseudocode of the HMODPSO algorithm.

TABLE 1: The average results of the Pareto optimal solutions.

\begin{tabular}{|c|c|c|c|c|c|c|}
\hline Pareto solutions & NSGA-II & MODPSO & MODPSO-GSA & HMODPSO-1 & HMODPSO-2 & HMODPSO-3 \\
\hline 13 & $*$ & $*$ & 5.0500 & 5.0524 & 5.0524 & 5.0524 \\
\hline 14 & * & * & 5.0779 & 5.0998 & 5.1052 & 5.1054 \\
\hline 15 & 5.0551 & 5.0550 & 5.1516 & 5.1510 & 5.1536 & 5.1576 \\
\hline 16 & 5.1220 & 5.0635 & 5.1971 & 5.1975 & 5.1976 & 5.1980 \\
\hline 17 & 5.1346 & 5.1306 & 5.2380 & 5.2372 & 5.2382 & 5.2291 \\
\hline 18 & 5.2158 & 5.2031 & 5.2682 & 5.2639 & 5.2680 & 5.2682 \\
\hline 19 & 5.2375 & 5.2283 & 5.2894 & 5.2876 & 5.2899 & 5.2904 \\
\hline 20 & 5.2637 & 5.2614 & 5.3038 & 5.3095 & 5.3135 & 5.3183 \\
\hline$T / \mathrm{s}$ & 56.23 & 3.1364 & 6.8735 & 4.4328 & 8.7426 & 12.5279 \\
\hline
\end{tabular}

TABLE 2: The average results of the Pareto optimal solutions.

\begin{tabular}{lc}
\hline Pareto solutions & HMODPSO-1 \\
\hline 3 & 1.7280 \\
4 & 1.7856 \\
5 & 1.8372 \\
6 & 1.8712 \\
\hline$T / \mathrm{s}$ & 1.1155 \\
\hline
\end{tabular}

DWTA until no targets or weapons are left in the cooperative air combat.

\section{Conclusions}

This paper has presented a new hybrid multiobjective DPSO called HMODPSO algorithm to solve the cooperative air combat DWTA multiobjective optimization problems. The proposed algorithm has three advantages. (a) The leader particle selecting operator and neighborhood searching operator can improve the search ability and the rate of convergence. (b) The repairing operator can enhance the efficiency of generating feasible solutions. (c) The Cauchy mutation operator can boost the diversity and distribution of Pareto optimal solutions. HMODPSO can find solutions with good accuracy, convergence, and diversity, which can generate obviously better DWTA decisions without the cost of overmuch extra computation time compared to NSGAII, MODPSO, MODPSO-GSA, and MOEA/D. HMODPSO can also improve the cooperative air combat effectiveness efficiently.

Future research will focus on optimizing DWTA instances under larger scales. In addition, more new mechanisms on reducing the time complexity of the proposed algorithm will also be investigated. 


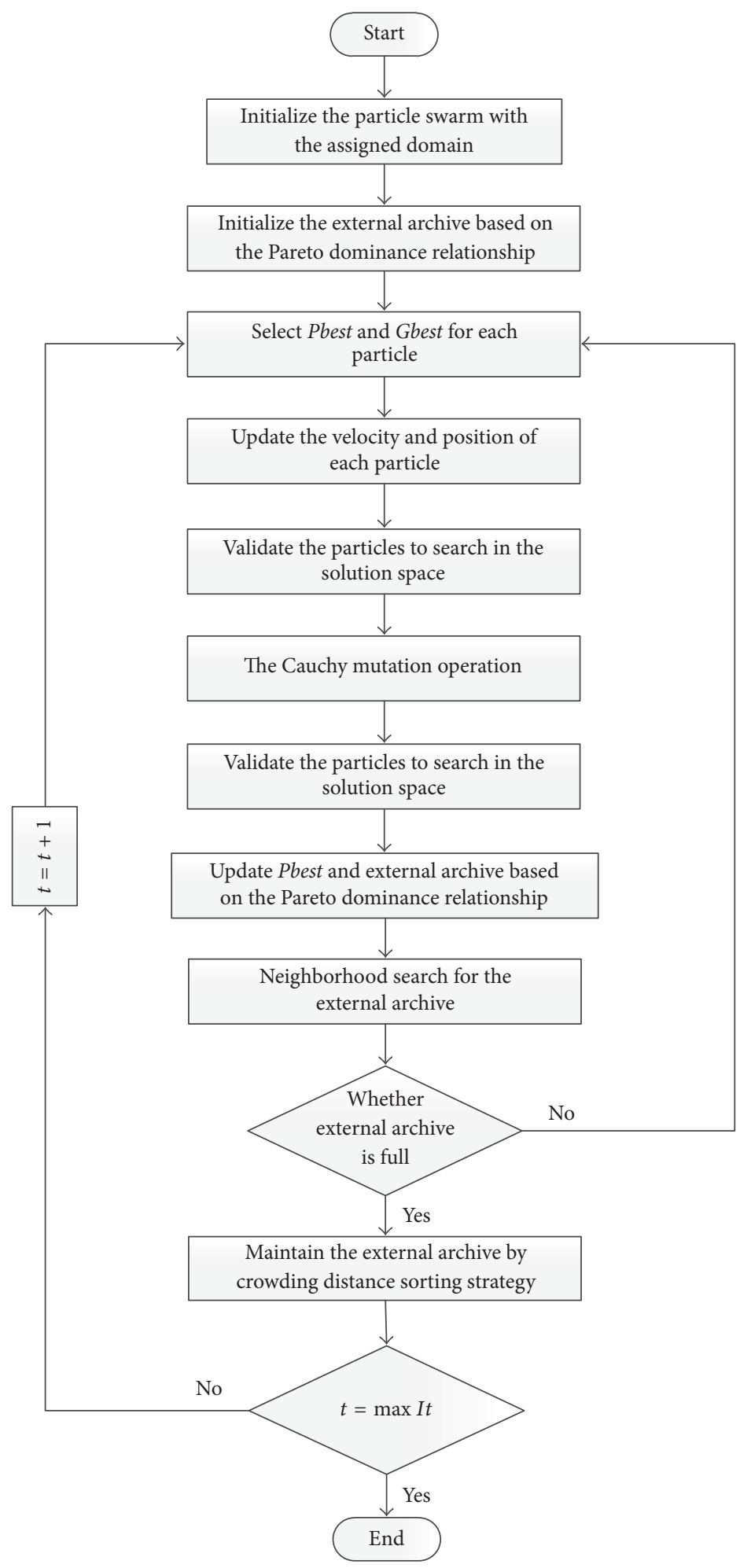

Figure 4: The entire flowchart of HMODPSO. 


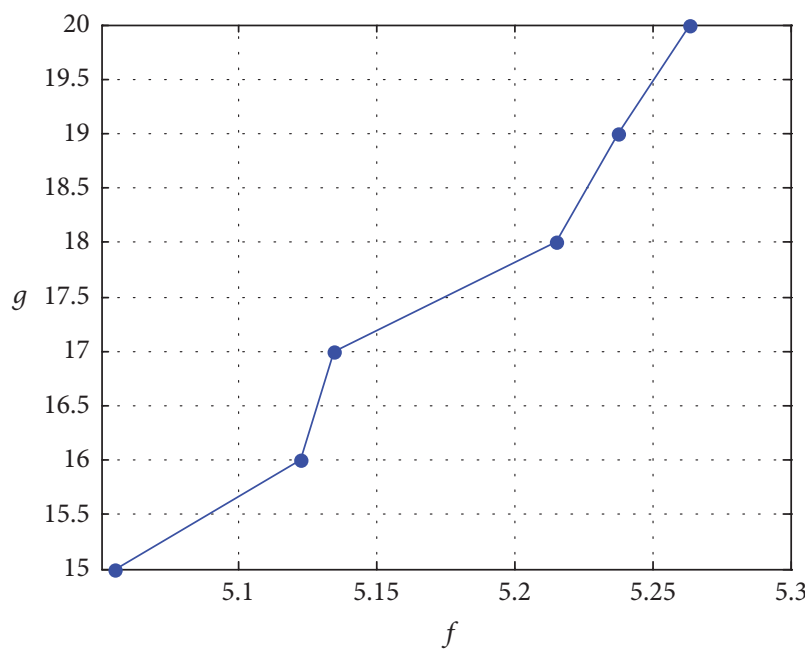

(a) NSGA-II

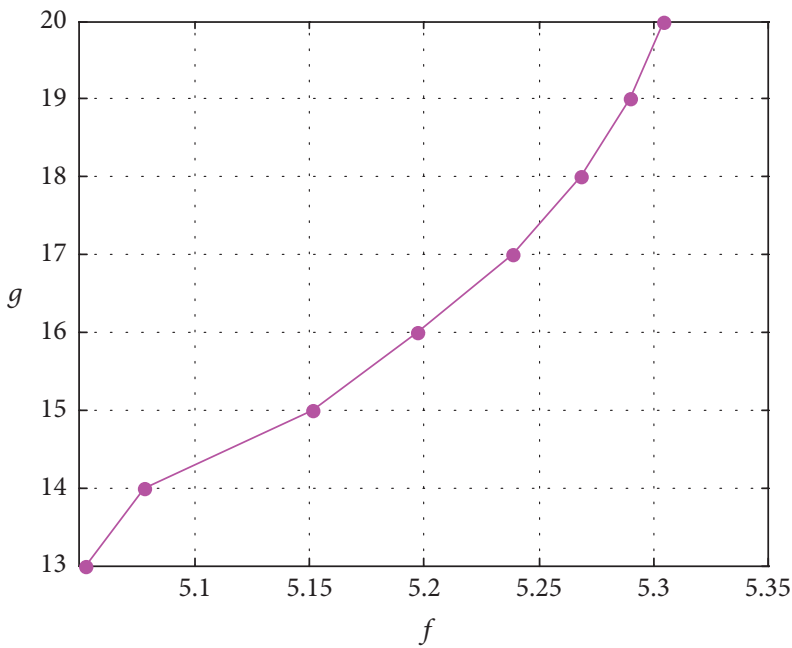

(c) MODPSO-GSA

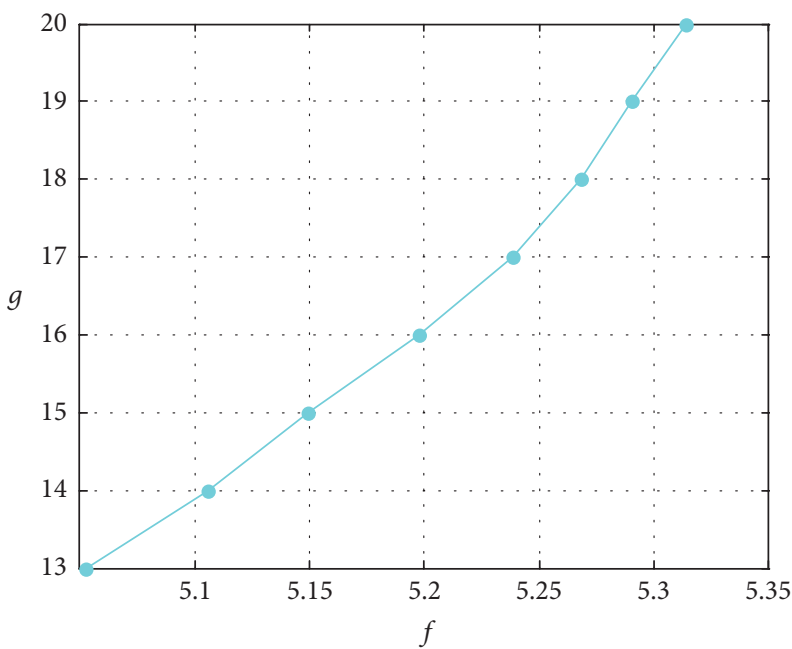

(e) HMODPSO-2

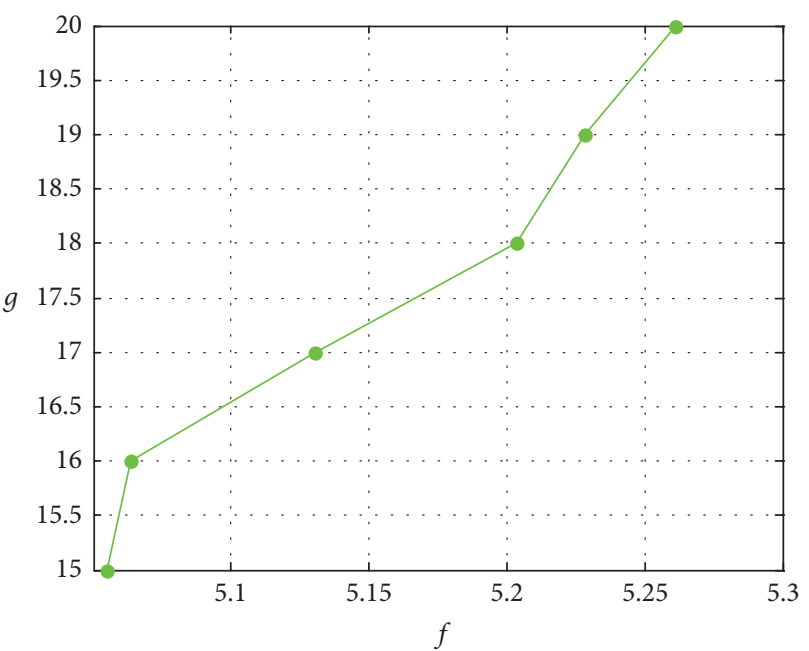

(b) MODPSO

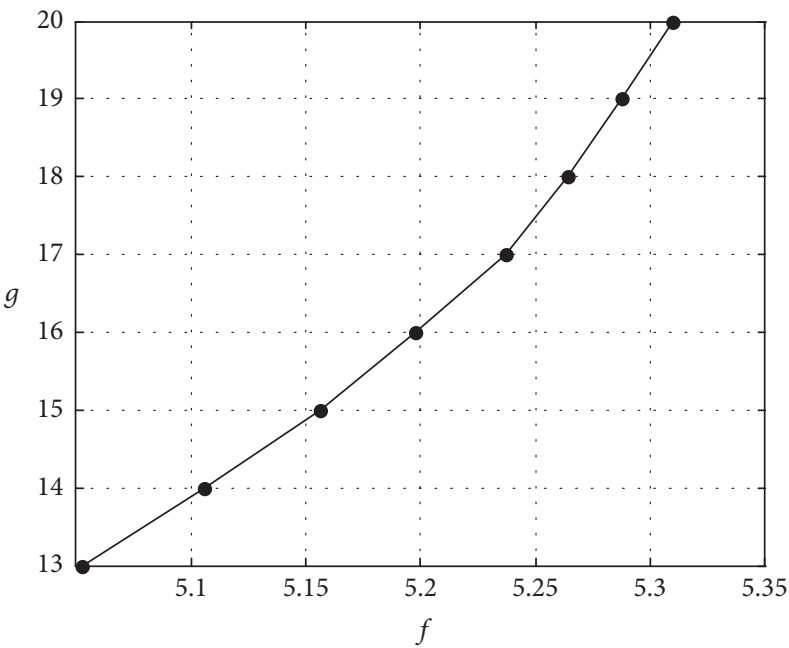

(d) HMODPSO-1

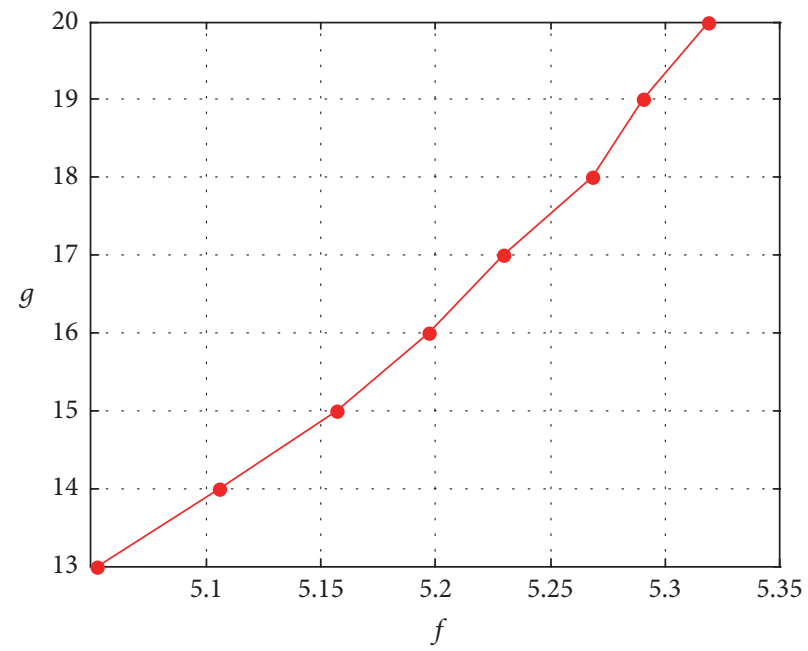

(f) HMODPSO-3

FIgUre 5: The Pareto fronts obtained by six algorithms. 


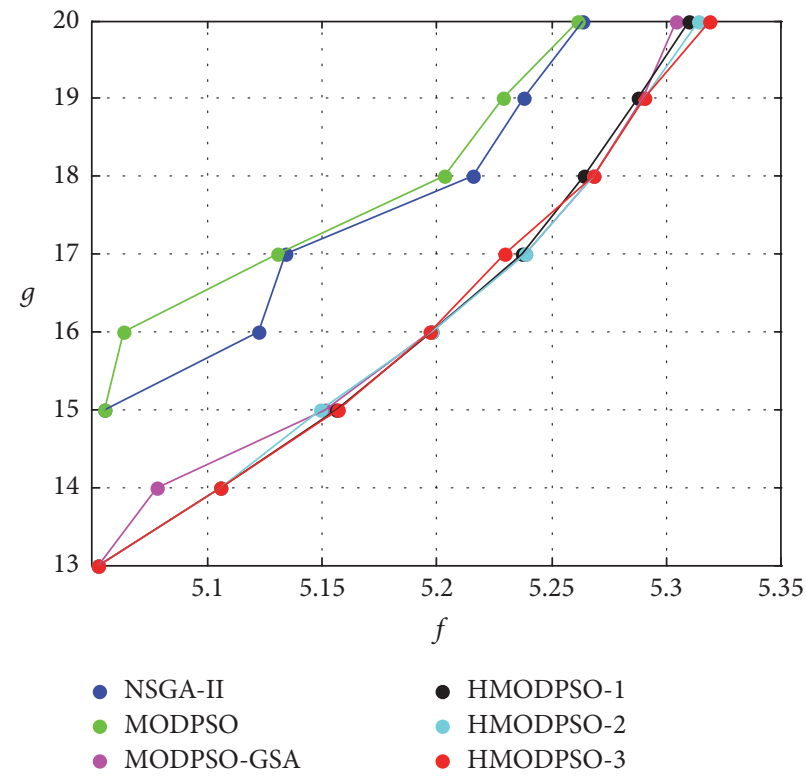

FIgURE 6: The compared Pareto fronts.

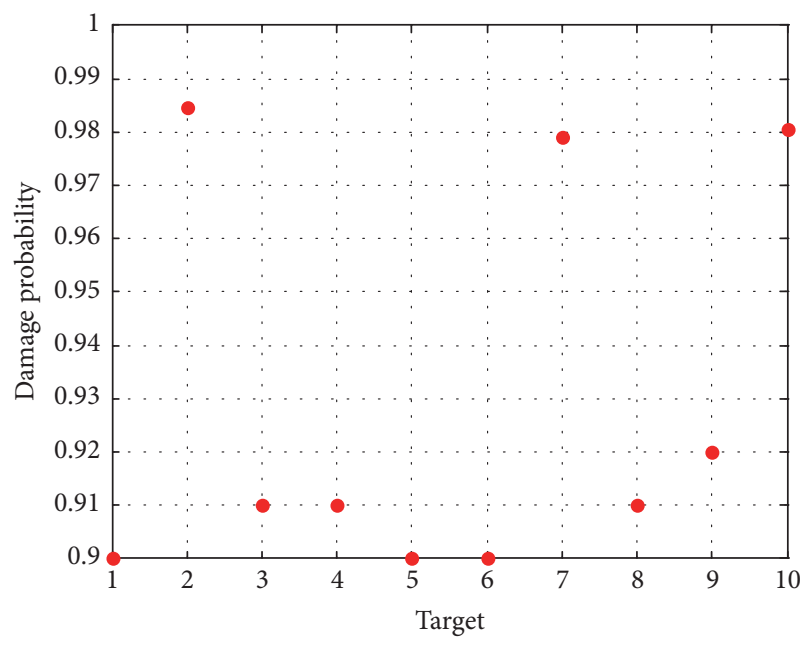

(a) The damage probability

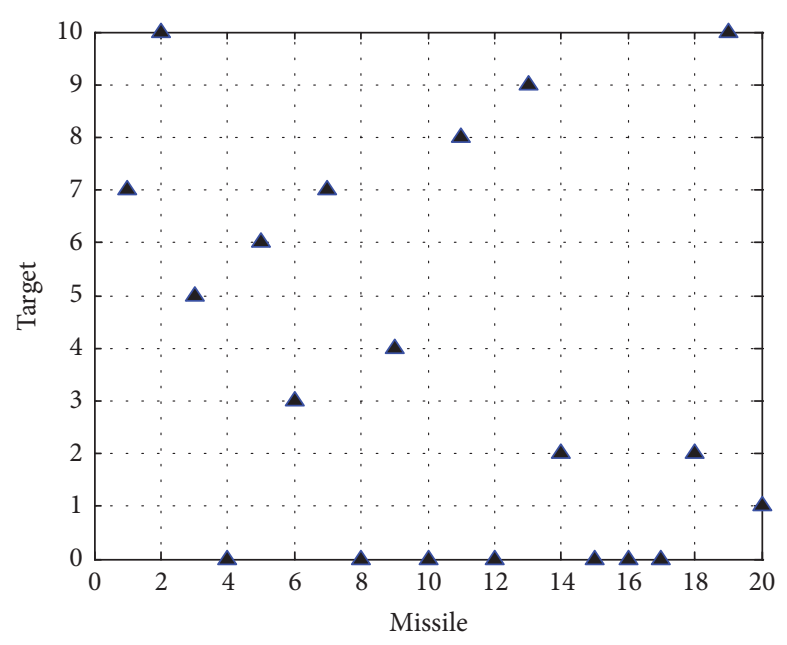

(b) The scheme

FIgURE 7: The WTA scheme obtained by HMODPSO-1.

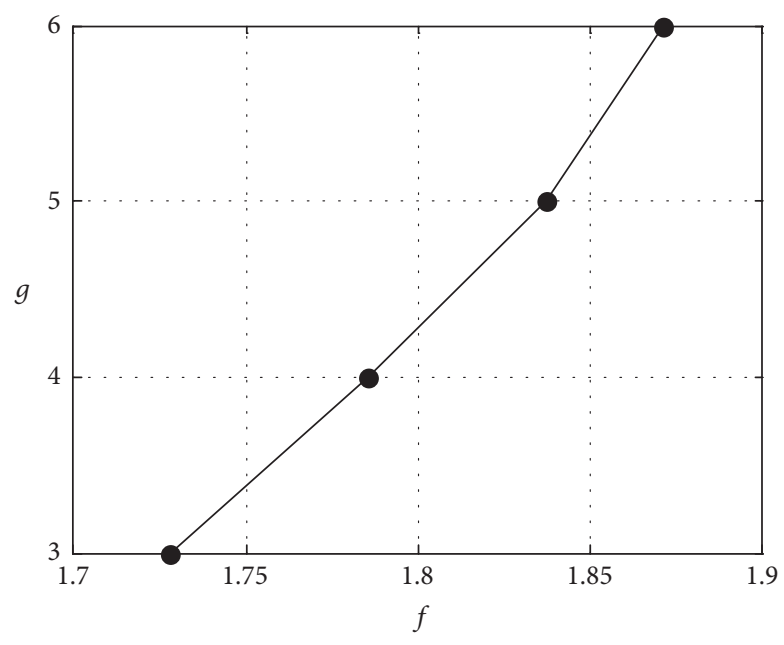

FIgURE 8: The Pareto front obtained by HMODPSO-1. 


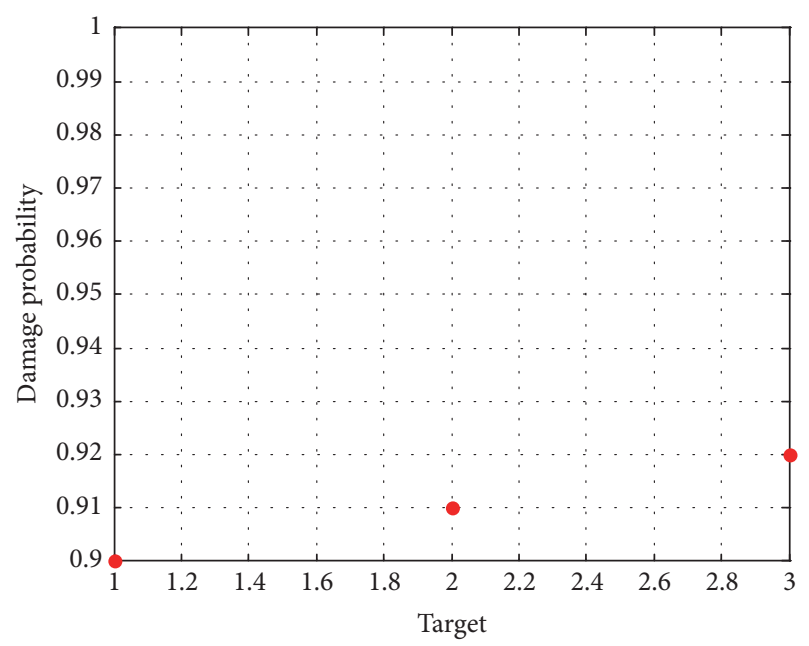

(a) The damage probability

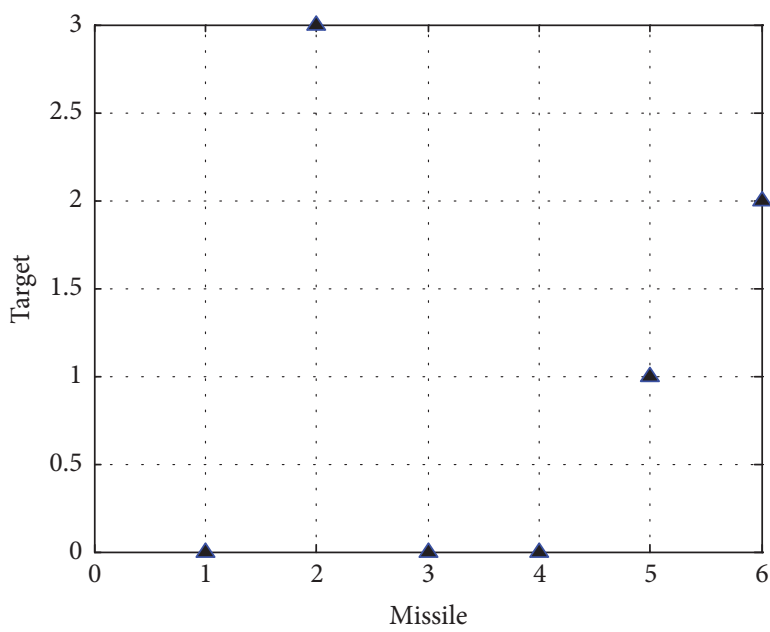

(b) The scheme

FIgURE 9: The WTA scheme obtained by HMODPSO-1.

\section{Conflicts of Interest}

The authors declare that they have no conflicts of interest.

\section{References}

[1] S. P. Lloyd and H. S. Witsenhausen, "Weapons allocation is NPcomplete," in Proceedings of the IEEE Summer Simulation Conference, pp. 1054-1058, Reno, Nev, USA, 1986.

[2] P. A. Hosein and M. Athans, "Preferential defense strategies. Part I: the static case," Tech. Rep. LIPS-P-2002, 1900.

[3] P. A. Hosein and M. Athans, "Preferential defense strategies. Part II: the dynamic case,” Tech. Rep. LIPS-P-2003, 1900.

[4] H. Cai, J. Liu, Y. Chen, and H. Wang, "Survey of the research on dynamic weapon-target assignment problem," Journal of Systems Engineering and Electronics, vol. 17, no. 3, pp. 559-565, 2006.

[5] D. Khosla, "Hybrid genetic approach for the dynamic weapontarget allocation problem," in Battlespace Digitization and Network-Centric Warfare, vol. 4396 of Proceedings of SPIE, pp. 244-259, August 2001.

[6] C. B. Liu, H. Y. Wang, and Z. M. Qiu, "An adaptive Memetic algorithm solving dynamic weapon target assignment problem," in Proceedings of the in 2nd International Conference on Information Engineering and Computer Science, pp. 1-4, Wuhan, China, 2010.

[7] J. Chen, B. Xin, Z. H. Peng, L. H. Dou, and J. Zhang, "Evolutionary decision-makings for the dynamic weapon-target assignment problem," Science in China Series F: Information Sciences, vol. 52, no. 11, pp. 2006-2018, 2009.

[8] B. Xin, J. Chen, J. Zhang, L. H. Dou, and Z. H. Peng, "Efficient decision-makings for dynamic weapon-target assignment by virtual permutation and tabu search heuristics," IEEE Transactions on Systems, Man, and Cybernetics, Part C, vol. 40, no. 6, pp. 649-662, 2010.

[9] B. Xin, J. Chen, Z. Peng, L. Dou, and J. Zhang, "An efficient rulebased constructive heuristic to solve dynamic weapon-target assignment problem," IEEE Transactions on Systems, Man, and
Cybernetics Part A:Systems and Humans, vol. 41, no. 3, pp. 598606, 2011.

[10] Y. Wang, J. Li, W. Huang, and T. Wen, "Dynamic weapon target assignment based on intuitionistic fuzzy entropy of discrete particle swarm," China Communications, vol. 14, no. 1, pp. 169179, 2017.

[11] Y. Wang, W. G. Zhang, and Y. Li, "An efficient clonal selection algorithm to solve dynamic weapon-target assignment game model in UAV cooperative aerial combat," in Proceedings of the 35th Chinese Control Conference (CCC '16), pp. 9578-9581, IEEE, Chengdu, China, July 2016.

[12] X. Liu, Z. Liu, W.-S. Hou, and J.-H. Xu, "Improved MOPSO algorithm for multi-objective programming model of weapontarget assignment," Systems Engineering and Electronics, vol. 35, no. 2, pp. 326-330, 2013.

[13] D. Zhou, X. Li, Q. Pan, K. Zhang, and L. Zeng, "Multiobjective weapon-target assignment problem by two-stage evolutionary multiobjective particle swarm optimization," in Proceedings of the IEEE International Conference on Information and Automation (ICIA '16), pp. 921-926, Ningbo, China, August 2016.

[14] D. P. Lötter and J. H. Van Vuuren, "A tri-objective, dynamic weapon assignment model for surface-based air defence," ORiON, vol. 32, no. 1, pp. 1-22, 2016.

[15] J. Li, J. Chen, B. Xin, and L. Dou, "Solving multi-objective multistage weapon target assignment problem via adaptive NSGAII and adaptive MOEA/D: a comparison study," in Proceedings of the IEEE Congress on Evolutionary Computation (CEC '15), pp. 3132-3139, Sendai, Japan, May 2015.

[16] J. Li, J. Chen, B. Xin, L. H. Dou, and Z. H. Peng, "Solving the uncertain multi-objective multi-stage weapon target assignment problem via MOEA/D-AWA," in Proceedings of the IEEE Congress on Evolutionary Computation, pp. 4934-4941, Vancouver, Canada, 2016.

[17] J. Kennedy and R. Eberhart, "Particle swarm optimization," in Proceedings of the IEEE International Conference on Neural Networks, pp. 1942-1948, Perth, Australia, 1995.

[18] M.-F. Leung, S.-C. Ng, C.-C. Cheung, and A. K. Lui, "A new strategy for finding good local guides in MOPSO," in Proceedings of the IEEE Congress on Evolutionary Computation (CEC '14), pp. 1990-1997, IEEE, Beijing, China, July 2014. 
[19] J. Yan, X. M. Li, L. J. Liu, and F. X. Zhang, "Weapon-target assignment based on Memetic optimization algorithm in beyond-visual-range cooperative air combat," Journal of Beijing University of Aeronautics and Astronautics, vol. 40, no. 10, pp. 1424-1429, 2014.

[20] G. Peng, Y. W. Fang, D. Chai, Y. Xu, and W. S. Peng, "Multiobjective particle swarm optimization algorithm based on sharing-learning and Cauchy mutation," in Proceedings of the 35th Chinese Control Conference, pp. 9155-9160, Chengdu, China, 2016.

[21] Y.-C. Wang, G.-L. Shan, and J. Tong, "Solving sensor-target assignment problem based on cooperative memetic PSO algorithm," Systems Engineering and Electronics, vol. 35, no. 5, pp. 1000-1007, 2013.

[22] C. R. Raquel and P. C. Naval, "An effective use of crowding distance in multiob-jective particle swarm optimization," in Proceedings of the 7th Annual Conference on Genetic and Evolutionary Computation, pp. 257-264, Washington, DC, USA, 2005.

[23] K. Deb, A. Pratap, S. Agarwal, and T. Meyarivan, "A fast and elitist multiobjective genetic algorithm: NSGA-II," IEEE Transactions on Evolutionary Computation, vol. 6, no. 2, pp. 182-197, 2002.

[24] J. Gu, J. Zhao, J. Yan, and X. Chen, “Cooperative weapon-target assignment based on multi-objective discrete particle swarm optimization-gravitational search algorithm in air combat," Journal of Beijing University of Aeronautics and Astronautics, vol. 41, no. 2, pp. 252-258, 2015. 


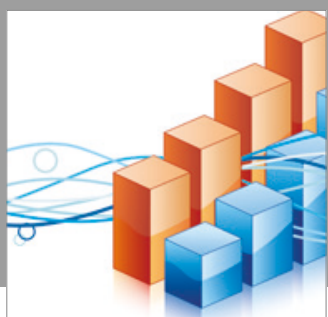

Advances in

Operations Research

vatersals

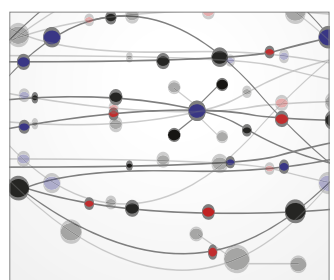

\section{The Scientific} World Journal
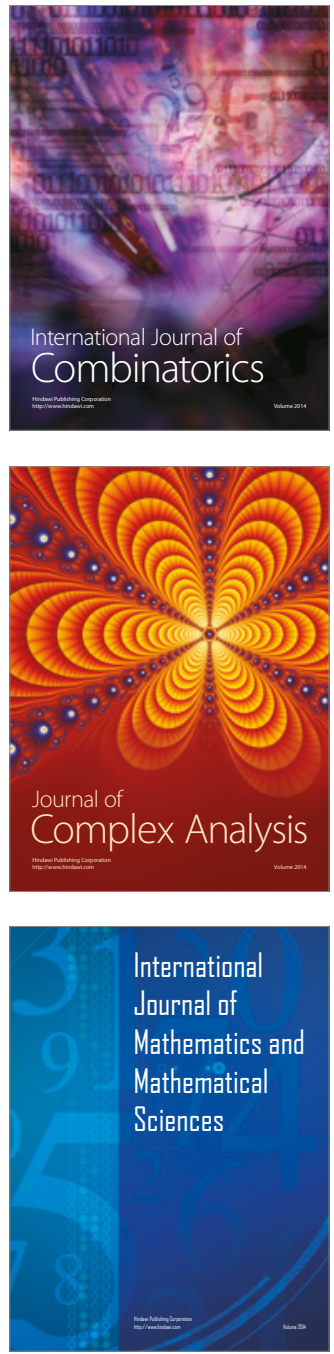
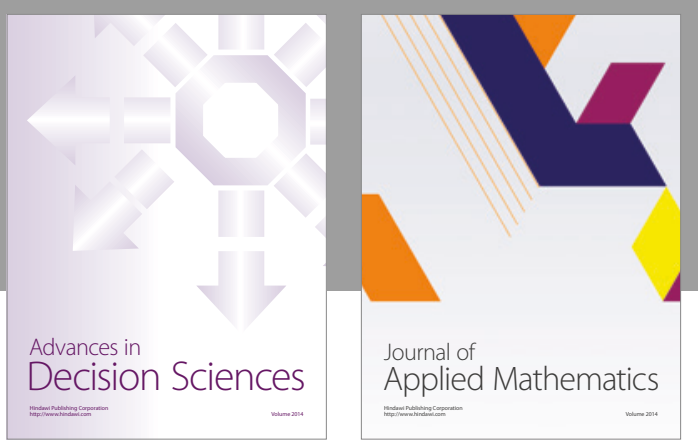

Algebra

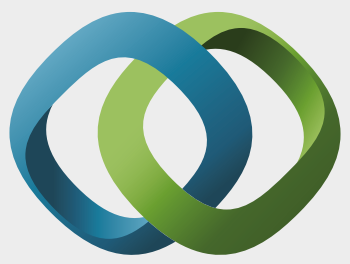

\section{Hindawi}

Submit your manuscripts at

https://www.hindawi.com
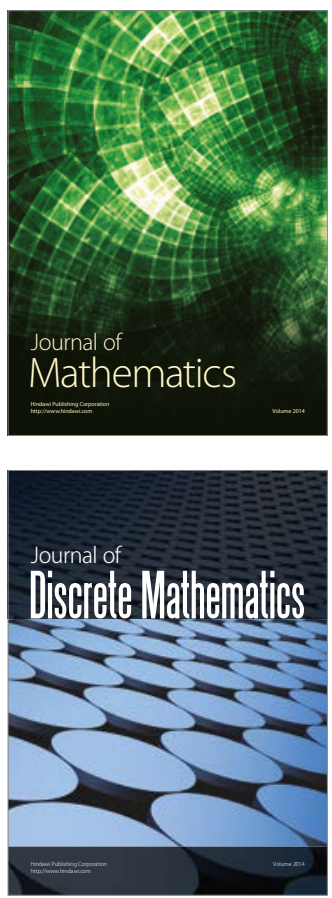

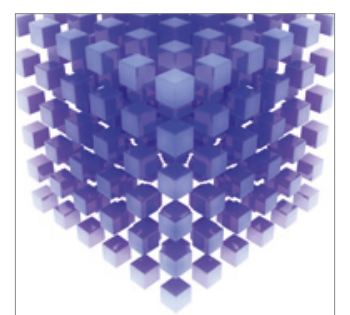

Mathematical Problems in Engineering
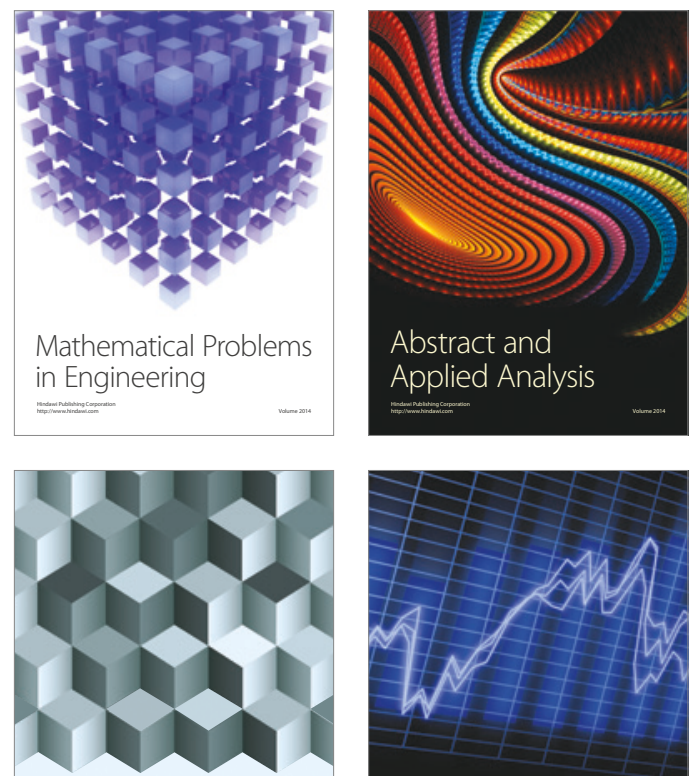

Journal of

Function Spaces

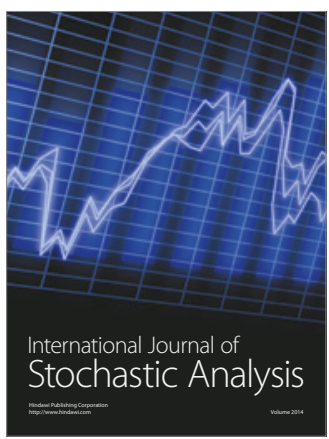

Probability and Statistics
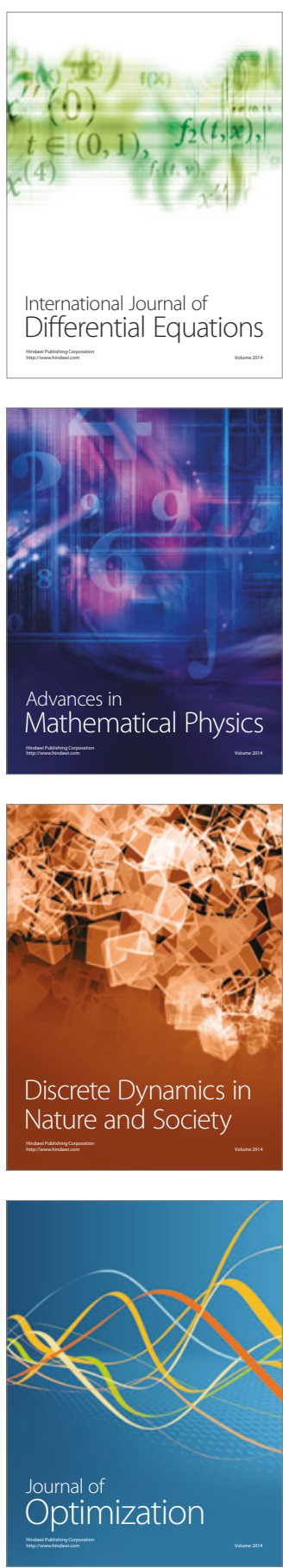